\title{
Der Islam und die Rechtsordnung in Ungarn im Mittelalter und im modernen Zeitalter
}

The Islam and the Legal Order in Hungary in the Midle Ages and in the Modern Age

The Islamic religion (hanafite rite), by virtue of Act nr. XVII adopted unanimously by both Houses (Lower House and Upper House i.e. Senate) of the then existing bicameral Hungarian Parliament on March 30, 1916 in the Kingdom of Hungary (regnum Hungariae) was given the status of a "recognized" religion i.e. religious community (in Latin: "licita religio"). By virtue of this Act/Law the Islamic religion got privileged legal status as well as legal protection. The status of the adherents of the Islamic religion became regulated, albeit not in a comprehensive way, in the sources of the legal order of the Kingdom of Hungary during the Midle Ages.

Keywords: Act nr. XVII of 1916 - Bosnia-Herzegovina - Bulla Aurea (Golden Bull) - hanafite rite - recognized religion (religio licita) - Treaty of Bereg of 1231 - Tripartitum of Werbőczy

Es steht fest, dass die Beziehung des Ungarntums und Ungarns zum Islam in eine Jahrtausende alte Vergangenheit zurückreicht. In der ungarischen Rechtsgeschichte werden diesbezüglich drei voneinander klar abgrenzbare Epochen unterschieden: I. von den Anfängen bis Ende des 13. Jahrhunderts, II. von 1541 bis 1687 und III. von 1878 bis 1918. Im Folgenden sollen diese Abschnitte eingehender erläutert werden.

\section{1.}

In der Zeit vor der ungarischen Landnahme, während der Völkerwanderung, begegneten die Ungarn auf dem weitläufigen Gebiet zwischen Wolga und Karpaten zweifelsohne Ethnien islamischer Religion oder Glaubens. Wie Konstantin Porphyrogenitus schreibt, schloss sich in Lebedia (in der Gemeinsprache Lewedien genannt, das Gebiet zwischen den Flüssen Don und Dnjepr) den sieben wandernden ungarischen Stämmen ein kabarischer Stamm an, der sich danach gemeinsam mit den Ungarn im Karpatenbecken niederließ (ca. 895-900 n. Chr.). ${ }^{1}$ Im Laufe der Landnahme trafen die ungarischen Stämme im Karpatenbecken erneut auf Ethnien islamischen Glaubens, auf Chasaren und Bulgaren. ${ }^{2}$ Diese verschmolzen ebenso wie die Kabaren, die mit den Ungarn gemeinsam gekommen waren, allmählich mit dem Ungarntum, ${ }^{3}$ ihre Religion behielten sie allerdings mit großer Wahrscheinlichkeit bei. Die islamische Bevölkerung östlicher Herkunft wird in den ungarischen Quellen (fontes) als „kálizok“ (die Kalizen) und "böszörmények" (ein türkischer Stamm) bezeichnet. ${ }^{4}$ Der Ausübung der islami-

\footnotetext{
${ }^{1}$ Zur Beziehung zwischen Ungarn und Muslimen siehe: SZÉKELY, Contacts entre Hongrois et Musulmans.

2 Zur Situation des Islam im mittelalterlichen Ungarn siehe: BALIC, Islam im mittelalterlichen Ungarn.

${ }^{3} \mathrm{Zu}$ den Beziehungen der ungarischen Sprache zur Arpadenzeit siehe: LigETI, A magyar nyelv.

${ }^{4}$ Der Begriff „káliz" kommt wahrscheinlich daher, dass sie aus Choresmien (oder Choresm) stammen.
} 
schen Religion legten die Ungarn ganz bis in die Zeit König Stephans [István] I. (997-1038) keine Steine in den Weg. König Stephan I. (der Heilige, Sanctus Stephanus) war selbstverständlich bemüht, auch die Bevölkerung islamischer Religion bzw. Konfession zu bekehren. Während jedoch die Bekehrung der Nicht-Christen zum Christentum alles in allem von Erfolg begleitet war, verhielt es sich mit den Muslimen anders. Sie kehrten nämlich mit aller Wahrscheinlichkeit sofort nach ihrer Taufe zu ihrem früheren Glauben und ihren Bräuchen (consuetudines) zurück. Daher gab es - wie zeitgenössische ungarische Gesetze bzw. königliche Dekrete (leges, decreta) belegen ${ }^{5}$ - sogar zwei Jahrhunderte nach König Stephan I. in Ungarn zahlreiche Gläubige islamischer Religion. Darüber aber, wie ihr Leben organisiert war, über welche Rechte sie verfügten, wissen wir nur wenig.

\section{2.}

Die ungarischen Gesetze (decreta bzw. leges) im 11. und 12. Jahrhundert bezeichneten die Muslime als "Ismaeliten“, seltener als „Sarazenen" (auf Lateinisch: ismaëlitae, saraceni). ${ }^{6}$ Gegenstand der Rechtsvorschriften, die sich auf sie

Die „kálizok” repräsentierten in der späten chasarischen Zeit die militärische Macht. Der Name „böszörmény" stammt mit persischer Vermittlung aus dem arabischen Wort musulmān, das über die Türken in die ungarische Sprache gelangte. Siehe: KARÁCSONYI, Kik voltak. Zur Herkunft der Muslime in Ungarn bietet der 1953 aufgetauchte Bericht des arabischen Reisenden Hāmid el-Granadino (1080/1081 - 1169 od. 1170) ebenfalls wertvolle Angaben, veröffentlicht von DUBLER, Abū Hāamid.

${ }^{5} \mathrm{Zu}$ den Gesetzen Stephans I. (des Heiligen) siehe aus der neueren Literatur: HAMZA, Szent István törvényei. ${ }^{6}$ Die Muslime wurden in erster Linie in den päpstlichen Briefen (epistolae) als Sarazenen bezeichnet. König Andreas II. benutzt die beiden Begriffe in einer seiner Schriften als Synonyme: „Saraceni, sive Ismaelitae". Siehe: CzEGLÉDY, Az Árpád-kori mohamedánokról. bezogen, war vor allem, wie sie zum christlichen Glauben zu bekehren seien. König Ladislaus [László] (1077-1095) verordnete in einem Dekret, dass Ismaeliten, die bereits getauft wurden und wieder zum Islam zurückgekehrt waren, von ihren früheren Wohnorten $\mathrm{zu}$ vertreiben und in anderen Dörfern anzusiedeln seien. König Koloman [Kálmán] (1095-1116) hingegen verfügte, dass dem König Bericht zu erstatten sei, wenn ein Ismaelit bei der Ausübung der Bräuche seiner früheren Religion ertappt wurde, und der Delator - die Person, die ihn angezeigt hatte - die Hälfte des Besitzes des „Schuldigen“ erhalte. Ebenfalls König Koloman ordnete an, dass jedes ismaelitische Dorf eine Kirche bauen solle und wenn diese fertiggestellt sei, solle die Hälfte der Bevölkerung des Dorfes ihren Wohnort verlassen und sich in einer anderen Gegend niederlassen. Weiterhin gab er die Anordnung, dass die Ismaeliten ihre Töchter nur mit Christen, das heißt mit Ungarn, verheiraten dürfen.

Die erwähnten Verfügungen hatten zum Ziel, die Ismaeliten mit den Christen zu vermischen, damit die religiöse Differenzierung unter der Bevölkerung des Landes auf diese Weise langfristig aufhöre. Trotz alledem gelang es nur, einen zahlenmäßigen Rückgang der Ismaeliten zu bewirken. Mit großer Wahrscheinlichkeit bemühten sich die ungarischen Herrscher im späteren Verlauf in Wirklichkeit auch nicht, die diesbezüglichen Gesetze allzu streng geltend zu machen. Daraus ergibt sich, dass Ungarn selbst im 13. Jahrhundert noch über eine vermutlich beachtliche Zahl an Einwohnern islamischen Glaubens verfügte. Wie aus zeitgenössischen Gesetzestexten (leges, decreta) hervorgeht, spielten sie in der Erledigung von finanziellen Angelegenheiten des Staates sogar eine bedeutende Rolle. ${ }^{7}$ Aufgrund des strengen kirchlichen Zinsverbots waren sie zu einem großen Teil die - um einen modernen Begriff zu gebrauchen - „Ban-

\footnotetext{
${ }^{7}$ Vgl. GÖNCZI, Ungarisches Stadtrecht 51.
} 
kiers" im damaligen Ungarn und verfügten somit über einen beachtlichen Einfluss auf dem Gebiet des Handels. ${ }^{8}$

Während die Dekrete (decreta) des 11. und 12. Jahrhunderts sie in religiöser Hinsicht betrafen, versuchten jene aus dem 13. Jahrhundert in erster Linie, ihren nicht als gering zu erachtenden Einfluss vor allem in der Finanzwelt einzuschränken. Die von König Andreas II. (12051235) 1222 erlassene Goldene Bulle (Bulla Aurea) verbietet ausdrücklich, dass staatliche Finanzbehörden Ismaeliten beschäftigen, zudem machte Andreas II. dem Klerus in dem 1233 ausgegebenen Abkommen von Bereg [auf Ungarisch: Beregi Egyezmény] bedeutende Zugeständnisse; ${ }^{9}$ die Kirche trat hier gegen die ismaelitischen Salzoffiziere auf, die ihre wirtschaftlichen Interessen verletzten. ${ }^{10}$

Von da an ging die Zahl der ismaelitischen Bevölkerung vermutlich kontinuierlich zurück, obschon es keine konkreten Angaben dazu gibt. Ein Grund dafür ist, dass sie den christlichen Glauben annahmen. Das „Tripartitum opus iuris consuetudinarii inclytae gentis Hungariae" (1541) von István Werbőczy erwähnt sie überhaupt nicht mehr, was die Schlussfolgerung erlaubt, dass in Ungarn zu Beginn des 16. Jahrhunderts Untertanen (subditi) islamischen Glaubens überhaupt nicht mehr oder aber nur in überaus geringer Zahl lebten.

\section{3.}

Einen erneuten Kontakt mit der islamischen Welt bedeuteten für die Ungarn die Kriege, die

\footnotetext{
${ }^{8}$ Den Reichtum der ungarischen Ismaeliten belegt beispielsweise, dass sie ihre Kinder in das ferne Jerusalem schickten, damit sie dort studierten. Siehe: KEDAR, Ungarische Muslime.

${ }^{9}$ Siehe vor allem $\S 7$ des Abkommens von Bereg.

${ }^{10}$ Zum Teil pachteten die Ismaeliten die Salinenregale, weswegen sie mit dem Klerus in Konflikt gerieten. Siehe: PAULINYI, A sóregále kialakulása.
}

das Land gegen die osmanischen Sultane führte. Diese Kriege nahmen wie bekannt ihren Anfang unter König Ludwig dem Großen aus dem Haus Anjou (1342-1382) und zogen sich mit einigen Unterbrechungen - man könnte sagen - ganz bis zum Ende des 18. Jahrhunderts hin. ${ }^{11}$

Unter dem Gesichtspunkt der Beziehungen sind das 16. und 17. Jahrhundert von besonderer Bedeutung. In den 40 Jahren nach der Schlacht bei Mohács im Jahre 1526 gelangte ein bedeutender Teil des Königreichs Ungarn (regnum Hungariae) unmittelbar unter osmanische Herrschaft. Fünfzehn Jahre nach der Schlacht bei Mohács, im Jahre 1541, eroberte Sultan Suleiman (1520-1566) Buda, die Hauptstadt des Landes, womit sich die osmanische Herrschaft auf dem Großteil Ungarns für 150 Jahre stabilisierte. Der größte Teil des Königreichs Ungarn, der die große Ebene entlang der Flüsse Donau und Theiß umfasste, wurde von den Osmanen erobert und damit Teil des Osmanischen Reiches. ${ }^{12}$

Das Königreich Ungarn und Siebenbürgen blieben - obschon Letzteres später zum osmanischen Vasallenstaat wurde - weitgehend frei vom Einfluss des Islam. In den besetzten Gebieten, die unmittelbar unter die Herrschaft des Sultans gelangt waren, war die Situation allerdings eine andere. Auf diesen Gebieten lagen die Verwaltung und die Rechtsprechung größ-

\footnotetext{
${ }^{11}$ Zur Beziehung zwischen Ungarn und dem Osmanischen Reich siehe umfassend, doch nicht mit dem Anspruch der Vollständigkeit: VÁRKONYI, Török világ; HEGYI, Egy világbirodalom végvidékén; FODOR, Magyarország és a török hódítás; HEGYI, Török berendezkedés Magyarországon.

${ }^{12} \mathrm{Zu}$ den gesellschaftlichen und wirtschaftlichen Verhältnissen des Osmanischen Reiches siehe umfassend: WITTEK, Rise of the Ottoman Empire; MILLER, Ottoman Empire; WERNER, Geburt einer Großmacht; INALCIK, Ottoman Empire; SHAW, History of the Ottoman Empire; KENT, The Great Powers; BENOISTMÉCHIN, Die Türkei; MANTRAN, L'Empire ottoman; INALCIK, Studies in Ottoman History; JORGA, Geschichte des Osmanischen Reiches; MAtuZ, Das Osmanische Reich.
} 
tenteils in der Hand der Eroberer; infolgedessen entstand ein Gebiet, auf dem - zumindest im Prinzip - das islamische Recht (sari'a) ${ }^{13}$ zum herrschenden Teil der Rechtsordnung wurde.

Nach Ansicht des namhaften Historikers Ferenc Salamon (1825-1892) wurde die ungarische Rechtsprechung auch im Eroberungsgebiet weitergeführt. ${ }^{14}$ Die Öffentlichkeit betrachtete es geradewegs als Verrat, wenn sich ein Ungar in einer Rechtsangelegenheit an einen Osmanen wandte. Dieses Verhalten - für das es im Ungarischen einen eigenen Begriff gab: „törökösség“ (auf Deutsch etwa „Türkenfreundlichkeit") galt als ein sui generis delictum, das eine schwere Strafe zur Folge hatte. ${ }^{15}$

Im Eroberungsgebiet existierte die frühere ungarische Staatsverwaltung zum Großteil nicht mehr; die Komitatsversammlungen (comitatus) hielten ihre Sitzungen auf dem Gebiet des Königreichs Ungarn ab. Die Osmanen hatten das eroberte Gebiet, das zum Nachteil des Königreichs Ungarn und des Fürstentums Siebenbürgen bis 1664 (dem Frieden von Vasvár) kontinuierlich expandierte, unter dem Gesichtspunkt der Verwaltung in "Paschaliks" aufgeteilt, an deren Spitze jeweils ein Pascha (eventuell ein Beglerbeg) stand. Anfangs gab es nur zwei Pa-

\footnotetext{
${ }^{13}$ Zur Geschichte des islamischen Rechts und seinerCharakteristika siehe aus der außerordentlich umfangreichen Literatur: GATTESCHI, Manuale; JUYNBOLL, Handbuch; JuYnBoll, Manuale; SANTILLANA, Istituzioni; MILLIOT, Introduction; TYAN, Institutions; FAYZEE, Outlines of Islamic Law; SCHACHT, Origins of Muhammadan Jurisprudence; TYAN, Méthodologie et sources; COUlSON, Histoire du droit islamique; PRITSCH, SPIES, Klassisches islamisches Recht; CHEHATA, Logique juridique; HouRANI, Basis of Authority; CheHATA, L'Equité; LinANT DE BELleFOnds, Traité de droit musulman; SCHACHT, Introduction au droit musulman; Noth, Verhältnis von Recht und Geschichte; Al-AzMeH, Islamic Law; Veramantry, Islamic Jurisprudence; CAPUTO, Introduzione al diritto Islamico; DURAND, Droit musulman; RAYNER, Theory of Contracts; G. MAKDISI, Religion, Law and Learning. 14 SALAMON, Magyarország a török hódítás korában.

${ }^{15}$ DEGRÉ, Négyeskönyv perjogi anyaga.
}

schaliks, Buda und Temesvár (Temeschwar, heute in Rumänien); ihre Zahl stieg später aber an, und in den letzten Jahrzehnten der Besetzung kamen die Paschaliks Eger, Nagykanizsa und Nagyvárad (Großwardein, heute in Rumänien) hinzu. Die Paschaliks wurden weiter in Sandschaks untergliedert, an deren Spitze die Sandschakbegs standen. ${ }^{16}$

Es gab im besetzten Gebiet keinen Unterschied zwischen Adel und Nicht-Adel, denn diesbezüglich machte die muslimische Welt eine gröBere gesellschaftliche und rechtliche Gleichberechtigung heimisch. Es erloschen damit auch jedwede Rechte und Verpflichtungen, die sich aus der alten ständischen Unterscheidung ergaben. Die im besetzten Gebiet gebliebenen Adeligen wurden ebenso „ráják" (Pächter) wie die Nicht-Adeligen und mussten die verschiedenen Steuern in gleicher Weise zahlen.

\section{4.}

Auf dem besetzten Gebiet durchlief das Privatrecht bedeutende Veränderungen. ${ }^{17}$ Es gab keinen Privatbesitz von Immobilien mehr, genauer gesagt ging gemäß den Prinzipien des muslimischen (islamischen) Rechts jede Immobilie in staatlichen Besitz über. Über jede Immobilie, über alle Einkünfte verfügte der Sultan, der Kalif, der diese sogleich in unmittelbare Verwaltung der Schatzkammer nahm oder seinen obersten Amtsträgern gegen Gebühren als Lehen (Khas-Besitz) zur Verfügung stellte. Selbstverständlich konnten die fraglichen Güter jederzeit zurückgenommen werden; des Weiteren war es nicht möglich, sie an die Nachkom-

${ }^{16}$ Zur Verwaltung des Eroberungsgebiets siehe: MATÚZ, A magyarországi török hódoltság néhány főbb sajátosságáról.

${ }^{17} \mathrm{Zu}$ den Merkmalen des ungarischen Privatrechts (ius privatum) im Mittelalter, insbesondere hinsichtlich der Einflüsse des römischen Rechts siehe: FöLDI, HAMZA, A római jog története $140 \mathrm{ff}$. 
men zu vererben. Damit ist die „Raubwirtschaft" der Paschas zu erklären, die dafür sorgte, dass das einst blühende wirtschaftliche Leben in dem besetzten Gebiet größtenteils verkümmerte.

Obgleich es, wie bereits erwähnt, im Eroberungsgebiet die vom Sultan verliehenen Immobilien betreffend keine Vererbung gab, konnte der Sohn eines Amtsträgers, der über ein Lehen verfügte, - wie aus schriftlichen Dokumenten bekannt ist - im Fall des Versterbens seines Vaters, wenn er noch minderjährig war oder sich in entsprechender Weise darum verdient gemacht hatte, Anspruch auf dessen Güter erheben. Die Schenkung konnte allerdings auch dann nur mit einem Schenkungsbrief (berat) des Sultans erfolgen. Das Erbrecht der im besetzten Gebiet lebenden Nicht-Adeligen blieb jedoch im Wesentlichen so, wie es zuvor gewesen war, da sie nach ungarischem Recht über recht wenig Immobilienbesitz verfügen konnten.

Zur Erledigung der Angelegenheiten bezüglich der Lehen befand sich in der Hauptstadt jedes Paschaliks eigens eine Behörde. Hier wurden auch die Rechnungsbücher der Staatsverwaltung geführt: die Defters. An der Spitze der Behörde stand der Defterdar, der die Arbeit der ihm unterstellten Finanzbeamten bzw. Finanzverwalter überwachte.

\section{5.}

Mit der Ablösung des ungarischen Verwaltungswesens erloschen die früheren Finanzorgane und das frühere Steuersystem. Die Osmanen führten eine neue Grundlage für das Steuerwesen ein, was mit einer ganzen Reihe von Steuern und anderen öffentlichen Einnahmen einherging. Den Großteil der Einkünfte der Schatzkammer stellten die Gebühren dar, die für die Nutzung der Flusshäfen und der Fähren zu zahlen waren, sowie die auf den Märkten gezahlten Standgebühren, der Marktzoll, die auf
Weinfässer erhobenen Steuern, die Besteuerung von Mühlen und Schlachtereien, weiterhin die Brautsteuer, die bei der Eheschließung gezahlt werden musste, und zwar bei den Muslimen an die Schatzkammer, bei den Christen hingegen an den Lehnsherrn. Es gab aber auch noch andere Steuergattungen, so beispielsweise die Kopfsteuer. Ausführliche Informationen $\mathrm{zu}$ alldem liefern die erhalten gebliebenen Defters. ${ }^{18}$

\section{6.}

Was die Gerichte im Eroberungsgebiet angeht, muss erwähnt werden, dass man zu Beginn für die Christen ein eigenes gerichtliches Forum einrichtete. Sultan Suleiman ernannte, als er 1541 Buda eroberte, István (Stephanus) Werbőczy (1458?-1541), den Verfasser des „Tripartitum opus iuris consuetudinarii inclyti regni Hungariae", den einstigen Palatin Ungarns, zum höchsten Richter der Christen. ${ }^{19}$ Dieses Amt verdankte seine Existenz jedoch allein der Tatsache, dass der Sultan die Person und die Rechtskenntnisse bzw. die Bewandertheit im Recht Werbőczys überaus schätzte. István Werbőczy war in Buda in diesem Amt auch tatsächlich tätig, wenngleich die osmanischen Beamten seiner Arbeit ständig Steine in den Weg legten. Die Institution war im Übrigen von nur sehr kurzer Dauer und erlosch noch in demselben Jahr (1541) mit dem Tod István Werbőczys.

Von dieser Zeit an befand sich die Gerichtsbarkeit, sowohl die Muslime als auch die Christen betreffend, ausschließlich in der Hand der os-

\footnotetext{
${ }^{18} \mathrm{Zu}$ den Defters und dem osmanischen Steuerwesen siehe: LÁsZlófALVI VeliCS, Magyarországi török kincstári defterek; KÁLDY NAGY, Harács-szedők és ráják; DERS., Magyarországi török adóösszeírások. ${ }^{19} \mathrm{Zu}$ Werbőczy siehe: KOLOZSVÁRI, ÓvÁRI, MÁRKUS, Werbőczy István Hármaskönyve; FRAKNóI, Werbőczy István életrajza. Aus der neueren Literatur siehe: HAMzA, Tanulmányok Werbőczy Istvánról; DERS., Werbőczy István .
} 
manischen Gerichte. Das Gerichtssystem gliederte sich folgendermaßen: In jeder Hauptstadt eines Sandschaks gab es einen Richter - den „Kadi“. Wenn ein Sandschak größer war, wurde er in Bezirke - in "Nahijes" - aufgeteilt. Jede Nahije besaß einen selbstständigen Unterrichter, einen Naib, der über eine rechtliche Ausbildung verfügte und der Stellvertreter des Kadis war. Beiden wurde das Amt von der Hauptstadt des Reiches, von Istanbul (Konstantinopel) aus verliehen.

Das größte Manko des osmanischen Verwaltungssystems waren die undefinierten Zuständigkeitsbereiche der einzelnen Behörden. Diese Unstrukturiertheit ist gerade bei den Gerichten am deutlichsten zu spüren. Der Kadi hätte eigentlich, betrachtet man streng genommen die Regeln des islamischen Rechts (sari'a), nichts anderes als die Aufgabe des Richters versehen dürfen. Nichtsdestotrotz verrichtete er außer seinen Aufgaben im bürgerlichen Recht, dem Strafrecht und in Vormundschaftsangelegenheiten auch notarielle Tätigkeiten und finanzielle Verwaltungsaufgaben.

\section{7.}

Im Folgenden wird der Wirkungskreis bzw. der Kompetenzbereich der Kadis in den ungarischen Gebieten, die unter osmanische Herrschaft gelangt waren, umrissen. In den Wirkungsreis (Kompetenzbereich) des Kadis gehörten vor allem Verfahren des bürgerlichen Rechts (ius privatum). Anzumerken ist jedoch, dass das Gericht des Kadis diesbezüglich nicht obligatorisch war. Die Christen wandten sich nur überaus selten an den Kadi, da die Gerichtskosten, die dem Kadi gezahlt werden mussten, meist sehr hoch waren. Da die Kosten immer von der obsiegenden Partei zu zahlen waren, bedeutete das für diese, wie gerecht der Fall auch war, einen riesigen Verlust. Somit wählten die Ungarn zur Klärung ihrer Streitsachen lieber ande- re Möglichkeiten. Verfahren bürgerlichen Rechts brachten eher nur Muslime vor den Kadi, die selbstverständlich nicht so erpressbar wie die Christen waren.

Vor den Kadi gehörten unter den Strafsachen in erster Linie Mord und Körperverletzung. Bei diesen beachtete der Kadi vor allem die Eintreibung der vom Gesetz als Strafe festgelegten Summen, die ihm selbst zustanden. War der Täter nicht auszumachen, so musste die Gemeinde zahlen, in der das Verbrechen geschehen war. Dies führte allmählich zu der Praxis, dass immer die Gemeinde die Bußgelder bezahlte. Um Ermittlung und Bestrafung kümmerte sich der Kadi nicht: Das war Aufgabe der Gemeinde beziehungsweise der Bauernkomitate, die infolgedessen gegenüber den Verbrechern Herren über Leben und Tod wurden. Wenn jedoch die Gemeinde einen Verbrecher als Strafe verstümmelte oder erhängte, musste sie erneut an den Kadi zahlen.

Auf Fälle der Ehrenkränkung und Verleumdung achtete der Kadi ebenfalls, doch nur wegen der im Gesetz vorgeschriebenen Bußgelder. Um die Überprüfung zu erleichtern, wurde später zur Praxis, dass der Kadi unter diesem Titel von den betroffenen Gemeinden eine jährliche Steuer eintrieb.

Obligatorisch gehörten die Nachlassangelegenheiten der „Ungläubigen“ vor den Kadi. Für die Nachlassverfahren war nämlich ein bestimmter Prozentsatz des Nachlasswertes zu zahlen, der dem Kadi zustand. Da gerade dies die hauptsächliche Einkommensquelle des Kadis war, überwachte er sorgsam, dass ihm die Todesfälle gemeldet wurden.

Ebenfalls vor den Kadi gehörten die Nachlassangelegenheiten der Muslime. Dies bedeutete für den Kadi jedoch eher eine Aufgabe der Finanzverwaltung, da der Nachlass der „Rechtgläubigen“ auf die Schatzkammer überging. Der Kadi hatte hierbei die Aufgabe, eine Inventur des Nachlasses zu erstellen, diesen zu einer ge- 
eigneten Zeit zu versteigern und die Einnahmen daraus für die Schatzkammer einzukassieren.

Der Kadi versah, wie bereits erwähnt, auch notarielle Funktionen. Gegen festgeschriebene Gebühren erstellte er Testamente, Heiratsurkunden und Verträge jeglicher Art. Die Gebühren, die er dafür einnahm, standen allerdings nicht ihm, sondern der Schatzkammer zu. Diese Tätigkeiten des Kadis nahmen vor allem die Muslime in Anspruch.

Schließlich war es auch Aufgabe des Kadis, die Eintreiber der Kopfsteuer zu bestimmen oder eventuell nach ursprünglichen Erhebungen die Kopfsteuer-Defters zu erstellen, des Weiteren die diesbezüglichen Abrechnungen zu überprüfen. Über all dies hinaus übte der Kadi das Aufsichtsrecht über die Moscheen, die Schulen, deren Stiftungen (wakf) sowie die hier angestellten Priester (Geistlichen) und Lehrer aus.

Was das Wirken des Kadis angeht, ist die ungarische Rechtsgeschichte betreffend der wichtigste Umstand, dass auf dem von Ungarn bewohnten Gebiet ein muslimisches Gerichtsorgan die Rechtsprechung ausübte, das sowohl in muslimischen als auch christlichen Angelegenheiten zuständig war und nach der Natur der Dinge immer das muslimische Recht anwendete.

Was das Religionswesen angeht, so ist aus erhalten gebliebenen Defters bekannt, dass die muslimischen Moscheen und Schulen im Eroberungsgebiet größtenteils aus staatlichen Geldern erbaut wurden, die Priester und Lehrer ihre Versorgung von der Schatzkammer erhielten. Eine Privatstiftung wurde zu diesem Zweck nur selten ins Leben gerufen.

In der Rangfolge der islamischen Priester (Geistlichen) müssen an erster Stelle die "Chatibs" erwähnt werden, die die feierlichen Freitagsgebete abhielten. Diese gab es nur in den größeren Städten, wo eine prunkvollere Moschee, eine "Dschami", stand. Dem Rang nach folgten ihnen die Imams, die die regelmäßigen Aufgaben eines Geistlichen versahen. Hiernach kamen die "Muaddins", die vom Turm der Moschee fünf- mal am Tag die vorgeschriebenen Gebete sangen.

Eng im Zusammenhang mit dem religiösen Leben stand die Bildung. Viele der Priester waren zugleich auch Lehrer. Es gab zwei Arten von Schulen: die "Mekteb" und die „Medrese“. Erstere war eine Volksschule, Letztere eine höhere Schule. Diejenigen, die eine Laufbahn als Priester, Richter oder im Verwaltungswesen einzuschlagen beabsichtigten, mussten auch die Medrese absolvieren, denn ohne diese Schule konnten sie kein Imam, Kadi oder "Sagird“ (Praktikant, Schreiber im Verwaltungswesen) werden.

Im Allgemeinen waren die Muslime gegenüber der christlichen Bevölkerung tolerant. Die Verfügung des Korans „In der Religion gibt es keinen Zwang“ kam im Allgemeinen zur Geltung. Und doch darf man den Humanismus der Osmanen nicht überschätzen, denn in der Toleranz spielte in vielen Fällen die Berechnung eine nicht zu vernachlässigende Rolle. Es kam vor, dass sie gegenüber den Katholiken für die Protestanten Partei ergriffen, ein anderes Mal nahmen sie protestantische Priester gefangen und ließen sie nur gegen Lösegeld frei. Es gab auch den Fall, dass sie Franziskanermönchen Schutzbriefe gaben, ein anderes Mal aber straften sie sie mit Stockschlägen. Sie behinderten die Renovierung christlicher Kirchen, ihre Ausbesserung; Tätigkeiten solcher Art waren immer von einer behördlichen Genehmigung abhängig.

Abgesehen von oben Genanntem kümmerten sie sich nicht viel um die religiösen Fragen der Christen. Unter anderem zeigt dies der Umstand, dass sie häufig auch Christen zur Überwachung finanzieller Angelegenheiten beschäftigten. Es kam häufig vor, dass städtische Richter oder einflussreiche Bürger als "Emins", als die Überwacher eines umfangreicheren Bereiches der Einnahmen und verantwortlich für die Abrechnungen, beschäftigt wurden, oder aber als „Amils“ (als Eintreiber für ein kleineres Objekt, zum Beispiel für den Torzoll). 


\section{8.}

Die anderthalb Jahrhunderte andauernde Türkenherrschaft übte auf das ungarische Recht beziehungsweise die Rechtsanwendung nur wenig Einfluss aus. Die Gemeinden behielten ihre früheren Einrichtungen bei, und die Bevölkerung lebte weiterhin nach dem früheren ungarischen Recht. Die osmanische Verwaltung kümmerte sich nicht weiter darum, sie achtete allein auf die genaue Einzahlung der Steuern.

Das Privatrecht blieb fast vollkommen gültig, vorausgesetzt es widersprach nicht den Grundprinzipien des osmanischen Staatssystems. Nur die ungarische Staatsmacht und das Verwaltungswesen übergaben ihren Platz den Osmanen. Es muss allerdings erwähnt werden, dass die untersten Einheiten, die Dörfer, Marktflecken und Städte ihre früheren charakteristischen Merkmale im Wesentlichen beibehielten.

Angemerkt werden sollte zudem, dass auch der Kontakt zwischen den Adeligen, die in das Gebiet des Königreichs Ungarn geflohen waren, und den im Eroberungsgebiet verbliebenen Leibeigenen nicht abbrach, sondern das Bewusstsein der Zusammengehörigkeit trotz der etwa anderthalb Jahrhunderte andauernden Türkenherrschaft von Generation zu Generation überliefert wurde. ${ }^{20}$ Es ist bekannt, dass der Adel (nobilitas), der aus den Komitaten im Eroberungsgebiet geflohen war, auf dem Gebiet des Königreichs ordentliche Komitatsversammlungen abhielt. Auch die Leibeigenen vergaßen die Adeligen nicht, sie betrachteten sie als ihre rechtmäßigen Herren und bemühten sich im Allgemeinen - sofern es ihnen möglich war -, auch ihren Verpflichtungen nachzukommen, die ihnen nach den ungarischen Gesetzen gegen-

\footnotetext{
${ }^{20}$ Unter anderem mit dieser Frage beschäftigt sich Ferenc Szakály. Siehe: SZAKÁLY, Magyar adóztatás a török hódoltságban; DERS., Magyar intézmények a török hódoltságban.
}

über den adeligen Großgrundbesitzern auferlegt waren.

9.

Der oben bereits erwähnte Umstand sowie die Antipathie, die sich gegenüber den osmanischen Eroberern aus den Jahrhunderte andauernden Kämpfen sowie den Heimsuchungen ergab, waren der entscheidende Grund dafür, dass die Spuren der muslimischen Herrschaft mit dem Abzug der Osmanen beinahe vollkommen verschwanden. Und ebenso verschwand auch das islamische Recht aus dem Land, ohne irgendeinen Einfluss auf die Entwicklung bzw. Fortbildung des ungarischen Rechts (ius patrium) ausgeübt zu haben. Eine große Rolle spielte dabei, dass gleichzeitig mit dem Abzug des osmanischen Militärs auch die islamischen Einwohner, die sich in den Städten niedergelassen hatten, Ungarn verließen und es nichts mehr gab, was ein Weiterbestehen des muslimischen Rechts in irgendeinem Maße begründet hätte.

Zusammenfassend lässt sich feststellen, dass ein Teil des Königreichs Ungarn durch die 150 Jahre andauernde Herrschaft der Osmanen zwar zum Herrschaftsgebiet des islamischen Rechts wurde, dieser Umstand aber keinerlei Einwirkung auf die Entwicklung des ungarischen Rechts hatte, keine Spur des Einflusses, der Wirkung der islamischen Religion im einheimischen ungarischen Recht (ius patrium) auffindbar ist.

\section{0.}

Eine neuere Epoche der Beziehung zwischen dem Ungarntum und der muslimischen Welt begann mit der Okkupation BosnienHerzegowinas (auf Türkisch: Bosna-Hersek) durch die Österreichisch-Ungarische Monarchie („Doppelmonarchie“) im Jahr 1878. Mit der Okkupation gelangte Ungarn als ein Teil der Doppelmonarchie gemeinsam mit Österreich an ein 
Gebiet, dessen Einwohner zum Teil islamischen Glaubens waren. Trotz allem brachte weder die Okkupation noch die 1908 erfolgte Annexion, als die Beziehung zwischen Bosnien-Herzegowina und der Österreichisch-Ungarischen Monarchie noch enger wurde, eine Veränderung einzelner Vorschriften im ungarischen Recht mit sich. Dies war schon alleine aus dem Grund nicht notwendig, da Bosnien-Herzegowina letztendlich nicht zu einem Gebiet bzw. Bestanteil des ungarischen Staates (regnum Hungariae) wurde.

Auch nach der Okkupation BosnienHerzegowinas durch Österreich-Ungarn blieb das islamische, genauer gesagt das in bedeutendem Maße islamische Elemente beinhaltende Privatrecht (Medschelle), das von weltlichen Gerichten und Scharia-Gerichten angewandt wurde, in Kraft. ${ }^{21}$ Die Sammlung Medschelle wurde in den Jahren 1869 und 1876 in 16 Bänden veröffentlicht. Zur Zusammenstellung dieser privatrechtlichen Sammlung (Kompilation) erhielt Pascha Ahmad Gawdat (1822-1895) im Jahr 1846 vom Großwesir Mustafa Reşid den Auftrag. Der Zweck der Kompilation war die „Europäisierung“, d. h. „Modernisierung“ des Privatrechts des Osmanischen Reiches. Die größtenteils auf den Lehren der hanafitischen Rechtsschule basierende, die sari'a als Grundlage betrachtende Kompilation folgt hinsichtlich ihrer Struktur, d. h. formal, den europäischen (west- und mitteleuropäischen) Kodex-Mustern, genauer gesagt dem Kodex-Modell. Die Medschelle wurde auch auf die Untertanen angewandt, die nicht islamischen Glaubens waren. ${ }^{22}$

${ }^{21}$ Zur Medschelle siehe aus der älteren Literatur dazu: DARESTE, Droit musulman; GRIGSBY, The Medjelle or Ottoman Civil Law.

${ }^{22}$ Zum in Bosnien-Herzegowina angewandten Recht siehe: EDUARD, Justizwesen Bosniens und der Herzegowina; PILAR, Entwicklungsgang; ZoBKOW, Anwendung des allgemeinen bürgerlichen Gesetzbuches; SCHOEN, Bosnisch-herzegowinisches, österreichisches, ungarisches und kroatisches Handelsrecht. Aus der jüngeren ungarischen Literatur siehe: HAMZA, Az
11.

Im Laufe des Ersten Weltkriegs wurde die islamische Religion, da das Osmanische Reich zum Verbündeten der Mittelmächte wurde, in dem Gesetzesartikel XVII aus dem Jahr 1916 zu einer gesetzlich anerkannten Konfession (,religio licita“) erklärt. Hier ist darauf $\mathrm{zu}$ verweisen, dass in Ungarn die Religionsgemeinde der Baptisten als erste im Jahre $1905 \mathrm{zu}$ einer anerkannten Konfession (auf Ungarisch: elismert felekezet) erklärt wurde. Allerdings muss auch betont werden, dass diese Anerkennung aufgrund der außenpolitischen Verpflichtung gegenüber dem Osmanischen Reich geschah; dabei spielte offensichtlich die perspektivische Möglichkeit des Anschlusses von Bosnien-Herzegowina zu Ungarn eine Rolle. Grund war also nicht die bei der Volkszählung 1910 nicht einmal tausend Personen (zahlenmäßig insgesamt 757!) erreichende Zahl ungarischer Untertanen islamischen Glaubens - inbegriffen das Nebenland Kroatien und Slawonien.

12.

Die Anerkennung der islamischen Religion bedeutete nicht, dass die Muslime ihre Religion zuvor nicht hätten frei ausüben können. Der Gesetzesartikel XLIII aus dem Jahr 1895 garantierte jedem die freie Ausübung seiner Religion, vorausgesetzt sie verstieß nicht gegen die staatliche und gesellschaftliche Ordnung. Der Gesetzesartikel XVII aus dem Jahr 1916 garantierte den Muslimen nur die Organisation und die Vereinigung in Form einer juristischen Person, was nach gültigem ungarischen Recht bloß den herkömmlichen und anerkannten Konfessionen zustand. Bis zum Inkrafttreten des Gesetzes

európai magánjog fejlődése 221f.; DERS., Développement du droit privé européen 66f.; DERS., Origine e sviluppo 337ff.; DERS., Entstehung und Entwicklung 493-499. 
konnten die Muslime ihre Religion nur unter strenger Überwachung, unter Aufsicht des Innenministers, ausüben. Dasselbe galt auch für ihre Religionsausübung im Rahmen eines Vereins. ${ }^{23}$

Entsprechend dem Gesetze, im Einklang mit dessen Vorschriften, gründeten die ungarischen Muslime in Budapest, d.h. in der Hauptstadt des regnum Hungariae eine Glaubensgemeinschaft. An ihrer Spitze stand als religiöser Anführer ein Imam, dessen Gehalt aus dem Staatshaushalt gedeckt wurde, und zwar auch nach dem Ersten Weltkrieg noch.

\section{3.}

Die Anerkennung des Islam ging nicht mit der Modifizierung der Regeln in Bezug auf die einzelnen Institute des ungarischen Rechts einher. Auch das Eherecht blieb unverändert, da die Anerkennung der muslimischen Konfession unter anderem - nicht die Möglichkeit einer Anerkennung der Polygamie bedeutete; sie bedeutete nicht, dass das ungarische Recht vom Prinzip der monogamen Ehe abgekommen wäre. Auch wurde die Verfügung des Strafgesetzbuches (Gesetzesartikel V aus dem Jahr 1878) nicht modifiziert, die die Bigamie zur Straftat (delictum) erklärte.

Im Hinblick darauf, dass die Anerkennung der islamischen Religion keine Abweichung von den allgemeinen Rechtsprinzipien (Rechtsgrundsätzen) bedeutete, gelangten auch nach der Verabschiedung des Gesetzesartikels XVII aus dem Jahr 1916 keine Elemente bzw. Institute des islamischen Rechts (sari'a) in das geltende ungarische Recht.

\footnotetext{
${ }^{23}$ Der Gesetzesartikel XVII war bis 1947 in Kraft.
}

\section{Korrespondenz:}

Univ.-Prof. Dr. Gábor HAMZA

„Eötvös Loránd“-Universität

Staats- und Rechtswissenschaftliche Fakultät

Egyetem tér 1-3

1053 Budapest, Ungarn

gabor.hamza@ajk.elte.hu

ORCID Nr. 0000-0002-6963-1201

\section{Abkürzungen:}

Siehe das allgemeine Abkürzungsverzeichnis: [http://www.rechtsgeschichte.at/files/abk.pdf]

\section{Literatur:}

A. Al-AzmeH (Hg.), Islamic Law. Social and Historical Contexts (London-New York 1988).

S. BALIC, Der Islam im mittelalterlichen Ungarn, in: Südost-Forschungen 23 (1964) 19-35.

J. BenOIST-MÉCHIN, Die Türkei 1908-1938. Das Ende des Osmanischen Reiches (Kehl am Rhein 1980).

G. CAPUTO, Introduzione al diritto Islamico I. I concetti generali - il madrimonio e la famiglia e successioni (Torino 1990).

C. CHeHATA, Logique juridique et droit musulman, in: Studia Islamica XXIII (1965) 5-26.

DERS., L' „Equité” en tant que source de droit hanafite, in: Studia Islamica XXV (1966) 123-138.

N.J. COULSON, Histoire du droit islamique (Paris 1995, ursprünglich: A History of Islamic Law, Edinburgh 1964).

K. CZEGLÉDY, Az Árpád-kori mohamedánokról és neveikről [Über die Mohammedaner der Arpadenzeit und ihre Namen], in: Nyelvtudományi Értekezések 70 [Material der II. Sprachwissenschaftlichen Konferenz, Budapest 1969] (Budapest 1970) 254-259.

R. DARESTE, Le droit musulman, in: Études d'histoire du droit (Paris 1889) 52-68.

A. DEGRÉ, Négyeskönyv perjogi anyaga [Das prozessrechtliche Material des Quadripartitum] (Budapest 1936).

C.E. Dubler (Hg.), Abū Hāmid el Granadino y su relación de viaje por tierras eurasiáticas. texto árabe, traducción e interpretación (Madrid 1959).

B. DURAND, Droit musulman. Droit successoral (Paris 1991). 
E. EDUARD, Das Justizwesen Bosniens und der Herzegowina (Wien 1889).

A.A. FAYZEE, Outlines of Islamic Law (Oxford ${ }^{3} 1964$ ).

P. FODOR, Magyarország és a török hódítás [Ungarn und die Eroberung durch die Osmanen] (Budapest 1991).

A. FöLDI, G. HAMZA, A római jog története és institúciói [Geschichte und Institutionen des römischen Rechts] (Budapest ${ }^{212016) .}$

V. FRAKNÓI, Werbőczy István életrajza [Die Biografie von István Werbőczy] (Budapest 1899).

D. Gatteschi, Manuale di diritto pubblico ottomano (Alexandria 1865).

K. GÖNCZI, Ungarisches Stadtrecht aus europäischer Sicht. Die Stadtrechtsentwicklung im spätmittelalterlichen Ungarn am Beispiel Ofen (Frankfurt am Main 1997).

W.E. GRIGSBY, The Medjelle or Ottoman Civil Law (London 1895).

G. HAmzA (Hg.), Au Tanulmányok Werbőczy Istvánról - Studien über István Werbőczy (Budapest 2001).

DERS., Szent István törvényei és Európa [Die Gesetze Stephans des Heiligen und Europa], in: G. HAMZA (Hg.), Szent István és Európa - Saint Étienne et l'Europe (Budapest 2001) 13-21.

DERS., Az európai magánjog fejlődése. A modern magánjogi rendszerek kialakulása a római jogi hagyományok alapján [Die Entwicklung des europäischen Privatrechts. Die Entwicklung der modernen privatrechtlichen Systeme auf der Grundlage des Römischen Rechts] (Budapest 2002).

DERS., Le développement du droit privé européen. Le rôle de la tradition romaniste dans la formation du droit privé moderne (Budapest 2005).

DERS., Entstehung und Entwicklung der modernen Privatrechtsordnungen und die römischrechtliche Tradition (Budapest 2009) 493-499.

DERS., Origine e sviluppo degli ordinamenti giusprivatistici moderni in base alla tradizione del diritto romano (Santiago de Compostela 2013).

DERS., Werbőczy István (1458?-1541) [István Werbőczy (1458?-1541)], in: G. HAMZA (Red.), Magyar jogtudósok - Ungarische Rechtsgelehrte, Bd. 5 (Budapest 2015) 9-22.

K. HegYI, Egy világbirodalom végvidékén [In der Grenzregion eines Weltreiches] (Budapest 1978).

DERS., Török berendezkedés Magyarországon [Das osmanische System in Ungarn] (Budapest 1995).

G.F. HOURANI, The Basis of Authority of Consensus in Sunnite Islam. Studia Islamica XXI (1965) 13-60.
H. INALCIK, The Ottoman Empire. The Classical Age 1300-1600 (London 1973).

DERS., Studies in Ottoman Social and Economic History (Oxford 1985).

N. JorGA, Geschichte des Osmanischen Reiches. Nach den Quellen dargestellt, 5 Bde. (Gotha 1908-1913, ND Frankfurt am Main 1990).

Th.W. JuYNBOLL, Handbuch des islamischen Gesetzes (Leiden 1910).

DERS., Manuale di diritto musulmano (Milano 1915).

Gy. KÁLDY NAGY, Harács-szedők és ráják. Török világ a XVI. századi Magyarországon [Requirierer und ráják (Pächter). Die osmanische Welt im Ungarn des 16. Jahrhunderts] (Budapest 1970).

DERS., Magyarországi török adóösszeírások [Osmanische Steuerregister in Ungarn] (Budapest 1970).

J. KARÁCSONYI, Kik voltak és mikor jöttek hazánkba a böszörmények vagy izmaeliták? [Wer waren und wann kamen die "böszörmények" oder Ismaeliten in unsere Heimat?], in: Értekezés a Történettudományok köréből XXIII 1, (1915) 14ff.

B.Z. KEDAR, Ungarische Muslime in Jerusalem im Jahre 1217, in: Acta Orientalia Academiae Scientiarum Hungariae XL (1986) 325-327.

M. KEnT (Hg.), The Great Powers and the End of the Ottoman Empire (Totowa 1979).

S. KOlozsvárI, K. ÓvÁRI, D. MÁRKUS, Werbőczy István Hármaskönyve [Das Tripartitum von István Werbőczy] (Budapest 1897).

A. LÁSZLÓFALVI VeLICS, Magyarországi török kincstári defterek [Die Defters der osmanischen Schatzkammer in Ungarn], Bd. 1. (Budapest 1886).

L. LigETI, A magyar nyelv török kapcsolatai a honfoglalás előtt és az Árpád-korban [Die Beziehungen der ungarischen Sprache zu den Turksprachen vor der Landnahme und in der Arpadenzeit] (Budapest 1986).

Y. LINANT DE BELLEFONDS, Traité de droit musulman comparé, 3 Bde. (Paris-La Haye 1965-1973).

G. MAKDISI; Religion, Law and Learning in Classical Islam (Oxford 1991).

R. MANTRAN, L'Empire ottoman du XVIe au XVIIIe siècle. Administration, économie, société. (Paris 1984).

J. MATÚZ, A magyarországi török hódoltság néhány főbb sajátosságáról [Einige Hauptmerkmale der osmanischen Besetzung in Ungarn], in: Gesta Hungarorum II. Történelmünk Mohácstól a kiegyezésig (Zürich 1985) 35ff.

DERS., Das Osmanische Reich. Grundlinien seiner Geschichte (Darmstadt 21990).

J.W. MiLLER, The Ottoman Empire and its Successors 1801-1927 (London ${ }^{31966) . ~}$ 
L. MiLLIOT, Introduction à l'étude du droit musulman (Paris 1953, ${ }^{2} 1987$ gemeinsam mit F.-P. BLANC).

A. Noth, Zum Verhältnis von Recht und Geschichte im Islam, in: Saeculum XXVI (1975) 341-346.

O. PAULINYI, A sóregále kialakulása Magyarországon [Die Entstehung der Salinenregale in Ungarn], in: Századok LVIII (1924) 627-647.

I. PILAR, Entwicklungsgang der Rezeption des österreichischen Allgemeinen Bürgerlichen Gesetzbuches in Bosnien und der Herzegowina unter besonderer Berücksichtigung des Immobilienrechtes, in: Festschrift zur Jahrhundertfeier des Allgemeinen Bürgerlichen Gesetzbuches, I. Teil (Wien 1911) 702-726.

E. PRITSCH, O. SPIES, Klassisches islamisches Recht, in: B Spuler (Hg.), Handbuch der Orientalistik, I. Abt.: Der Nahe und der Mittlere Osten, Erg.bd. III: Orientalisches Recht (Leiden-Köln 1964) 237-343.

S.E. RAYNER, The Theory of Contracts in Islamic Law (London ${ }^{51991) . ~}$

F. SALAMON, Magyarország a török hódítás korában [Ungarn im Zeitalter der türkischen Besetzung] (Pest 1864).

D. SANTILLANA, Istituzioni di Diritto Musulmano Malichita con riguardo anche al Sistema sciafiita, 2 Bde. (Roma 1925).

J. SCHOEN, Das bosnische-herzegowinische, österreichische, ungarische und kroatische Handelsrecht in ihren Verschiedenheiten (Wien 1913).

J. SCHACHT, The Origins of Muhammadan Jurisprudence (Oxford ${ }^{3} 1959$ ).
DERS., Introduction au droit musulman (Paris 1983 ursprünglich: An Introduction to Islamic Law, Oxford, 21966).

S.J. SHAW, History of the Ottoman Empire and Modern Turkey, 2 Bde. (New York 1976-77).

F. SZAKÁLY, Magyar adóztatás a török hódoltságban [Ungarische Besteuerung im osmanischen Eroberungsgebiet] (Budapest 1981).

DERS., Magyar intézmények a török hódoltságban [Ungarische Institutionen im osmanischen Eroberungsgebiet] (Budapest 1997).

Gy. SzÉKELY, Les contacts entre Hongrois et Musulmans aux IXe-XIIe siècles, in: G. KÁLDY-NAGY (Hg.), The Muslim East. Studies in Honour of Julius Germanus (Budapest 1974).

E. TYAN, Institutions du droit public musulman, Bd. 1 (Paris 1954).

DERS., Méthodologie et sources du droit islamique, in: Studia Islamica X (1959) 79-110.

Á.R. VÁRKONYI, Török világ és magyar külpolitika [Osmanische Welt und ungarische Außenpolitik] (Budapest 1975).

C.G. Veramantry, Islamic Jurisprudence. An International Perspective (Hongkong 1988).

E. Werner, Die Geburt einer Großmacht. Die Osmanen (1300-1481) (Wien 21972).

P. WiTTEK, The Rise of the Ottoman Empire (London 1939, ND London 1963).

M. ZOBKOW, Die Anwendung des allgemeinen bürgerlichen Gesetzbuches in Bosnien und der Herzegowina, in: Festschrift zur Jahrhundertfeier des Allgemeinen Bürgerlichen Gesetzbuches, I. Teil (Wien 1911) 727-752. 